\title{
Stem cells: technologies for the future
}

\author{
Margaret A. Goodell
}

BioTechniques 35:1232 (December 2003)

The last few years have witnessed an explosion of interest in stem cells, brought about by new findings on adult-derived stem cells and the successful isolation of human embryonic stem (ES) cells. Funding for stem cells studies has increased, and many new researchers have entered this field, formerly the province mainly of hematologists and a few developmental biologists. Moreover, a number of technologies have matured and can be applied to the study of these often rare cells, making it possible to generate new data more rapidly. In this special issue, six articles review some of the recent advances in stem cell research that will help move the field into the next era.

Adult stem cells are rare cells that replenish cells of their constituent tissue. While they are present in many adult tissues, they have been purified from very few, due to the lack of markers for their unique identification. In the bone marrow, they can now be fairly efficiently purified, but their paucity still makes it difficult to obtain sufficient quantities for many studies. For example, one can obtain about 50,000 stem cells from 10 mice in a full day's work. This number is sufficient for studies on their function, but not for many types of biochemistry experiments. Hence, research to identify genes that control stem cell activity has been slow. Recently, other technological advances have made it possible to apply more global approaches to the problem. $\mathrm{Li}$ and Akashi (page 1233) discuss the application of microarray technology to study gene regulation in stem cells.

One of the most important questions regarding stem cell regulation is the mechanism of self-renewal. What is the molecular decision that determines whether a daughter cell will become another stem cell or a progenitor committed to differentiate? The answer to this question has profound implications: in vitro expansion of adult stem cells for clinical use is a holy grail. Although we still do not have a definitive answer, studies in knock-out mice are beginning to chip away at this problem, as described by Pazianos and Reya (page 1240). Likewise, Sorrentino and colleagues (page 1248) explore the function of the multidrug resistance transporter, breast cancer resistance protein (BCRP). This protein has been implicated in the specific ability of stem cells to efflux drugs, as well as the dye Hoechst 33342 , which is widely used to purify hematopoietic stem cells.

While adult stem cell research has steamed full speed ahead, human ES cell research is the locomotive of the future: it has been slow starting, but is accelerating. Now that some of the regulatory issues have been clarified, many new researchers have been funded to work with them, and the stage is set for them to fully impact



Stem cells are multipotential cells that self-renew and give rise to lineage-restricted progenitors, which generate differentiated cell types. Over the past 4 years, advances in microarray technology, stem cell culture, murine xenotransplantation models, and gene knock-outs have all contributed to an enhanced understanding of stem cell regulation. These findings will facilitate development of new cell- and gene-based therapies, which will be applied to be human adult and embryonic stem cells. biomedicine. Chen, Lewis, and Kaufman (page 1253) describe the development and use of human ES cells, particularly with regard to their generation of hematopoietic cells.

A potential restraint to the clinical development of human ES-based technologies is the inability to test them in vivo. Hematologists have been struggling with this for decades and have developed a number of different mouse models that will support growth of various normal human cells. Some of these models, described by Nolta and coworkers (page 1262) are directly applicable to ES technology and to several cell and tissue types.

Of course the ultimate goal of all of this work is to improve human health. The gene therapy field has experienced both triumphs and setbacks in the last few years, and stem cell-based therapies will likely experience some of the same fits and starts. In a review, Spangrude (page 1273) examines some of the excitement of hematopoietic stem cell research and the greater implications for cell and gene therapy. This collection of reviews covers many of the most important recent developments in stem cell research and should offer something for the novice as well as the expert. 